\title{
SUPPLEMENTARY MATERIAL: PRACTICAL LARGE-SCALE SPATIO-TEMPORAL MODELING OF PARTICULATE MATTER CONCENTRATIONS
}

\author{
By Christopher J. Paciorek*, Jeff D. Yanosky*, Robin C. \\ Puett* ${ }^{*}$, Francine Laden*, and Helen H. SuH* \\ Harvard University, Harvard University, University of South Carolina, \\ Harvard University and Brigham and Women's Hospital, Harvard \\ University
}

\section{S1. Modelling alternatives.}

S1.1. Anisotropy, nonstationarity, and interactions. We plotted directional variograms for evidence of anisotropy in either the time-invariant or time-varying portions of the model. In the second-stage model, a directional variogram of residuals indicates more variability and longer spatial range in the east-west than the north-south direction, which makes sense in light of prevailing west to east winds and north-south gradients in pollution with more rural areas in the north of the study region. Directional variograms for the monthly data show similar patterns. Since the anisotropy appeared to be in the cardinal directions, we used gam() with tensor-product smooths of the $\mathrm{x}$ and $\mathrm{y}$ coordinates (Wood, 2006, p. 240) to allow the scaling of the coordinates to vary, but this approach did not improve predictive performance.

Given that there is less information for estimating the monthly spatial terms than the mean spatial term, $g_{\mu}(\cdot)$, we considered spatial nonstationarity in the context of the time-invariant portion of the model. To do this and also allow the smooth regression terms to vary regionally, we fit the second stage model separately for four subregions: (1) northern New England and northern New York; (2) southern New England and the mid-Atlantic region including eastern Pennsylvania; (3) the southern midwest, including western Pennsylvania; and (4) the northern midwest: Michigan and Wisconsin. For training, each region included a buffer of locations from neighboring regions to prevent boundary effects. The results showed a slight decrease

* Supported by EPA STAR R83-0545-010.

Keywords and phrases: additive model, air pollution, epidemiology, geoadditive model, smoothing, kriging, backfitting, stochastic EM 
in predictive performance relative to the core model for $\mathrm{PM}_{10}$, suggesting a bias-variance tradeoff, while for $\mathrm{PM}_{2.5}$, the results were very similar to the core model. There was some heterogeneity in covariate effects by region, but given the reduced sample sizes, it was not clear that these differences were robust.

Note that by fitting separate spatial models with different penalty estimates and different residual variances for each month in (3.1-3.2 in the main paper), we introduce nonstationarity in time into the model.

There are undoubtedly complicated interactions between the covariates, in particular with effects of covariates varying depending on the urbanness of the location, thereby introducing a nonstationarity between urban and rural areas. To allow nonparametric interactions between smooth regression terms, one can fit two-dimensional regression smooths (using gam() with tensor product bases to account for the different scales of the covariates). We interacted variables reflecting urbanness (land cover and the population density variables) with each of elevation and distance to the nearest road in the largest size class, two of the most important covariates, but the inclusion of the interactions did not improve predictive accuracy.

S1.2. Mixed model smoothing. To assess the sensitivity of the model to an alternative spatial smoothing approach, we considered the use of the $\operatorname{spm}()$ function in the SemiPar library in R, which fits penalized splines via a mixed model framework (Ruppert et al., 2003). We found that when fitting all the covariates in the second stage using $\operatorname{spm}()$, the model experienced singularities in the covariance structure. Therefore, we restricted our use of the mixed model approach to fitting the spatial residuals from models with the regression smooths fit via $\operatorname{gam}()$; this required backfitting in the second stage. The predictive accuracy for the mixed model spatial smoothing approach was worse than the core model with cross-validation $\mathrm{R}^{2}$ on the original scale of 0.579 compared to 0.618 for the $\mathrm{PM}_{10}$ model, and 0.721 compared to 0.771 for $\mathrm{PM}_{2.5}$. The mixed model approach used many fewer degrees of freedom for the time-invariant and time-varying spatial smooths, despite the use of a large number of knots.

S1.3. Kriging. Another possibility is to model the spatial structure using kriging. Here, we fit the regression terms using $\operatorname{gam}()$ and then fit the residuals using kriging based on maximum likelihood estimation of the covariance parameters, essentially an extended version of universal kriging. We fit simple isotropic kriging models for the residuals in each month in the first stage and for the spatial term in the second stage model. We use first order linear trend surfaces with the Matérn covariance with the differentiability 
parameter, $\nu \equiv 2$. For $\mathrm{PM}_{10}$, the model performance was very similar to the core model, but for $\mathrm{PM}_{2.5}$, model performance was worse, with a crossvalidation $\mathrm{R}^{2}$ of 0.727 for kriging compared to 0.771 for the core model. The kriging and core models attributed variability differently between the terms, with somewhat different regression smooths.

Some practical disadvantages of this approach are the large matrix manipulations that slow fitting and prediction and the need for backfitting in the second stage. Also, the presence of additional parameters in the model (spatial variance, spatial range, and trend parameters) compared to the single spline penalty parameter increases the possibility of getting stuck in local maxima during the numerical optimization. Note that a more sophisticated approach might borrow strength across months to more robustly estimate the covariance parameters for each month.

S2. Measurement error implications for health analysis. Gryparis et al. (2008) argue that spatial smoothing serves as a type of regression calibration that induces measurement error in the predictions of the Berkson type, rather than classical error. In our additive model, we have both spatial and regression smoothing, which should serve as a regression calibration for the predictions at new locations, conditioning on the covariates and spatial location. Under classical measurement error, the covariate, $X_{i}$, is measured with error as $W_{i}$, with the measurement error, $U_{i}$, independent of the covariate:

$$
\begin{aligned}
W_{i} & =X_{i}+U_{i} \\
X & \perp U \\
\operatorname{Var}(W) & =\operatorname{Var}(X)+\operatorname{Var}(U) .
\end{aligned}
$$

Under Berkson measurement error, the covariate, $X_{i}$, is instead centered around a proxy, $S_{i}$ (for smooth), with the measurement error independent of the proxy,

$$
\begin{aligned}
X_{i} & =S_{i}+V_{i} \\
S & \perp V \\
\operatorname{Var}(X) & =\operatorname{Var}(S)+\operatorname{Var}(V) .
\end{aligned}
$$

In our setting, the proxy is the prediction from the additive model. Under classical error, there is bias in both linear and nonlinear models, while under regression calibration and Berkson error, there is no bias in a linear model but precision of the estimates is worse than without any error. For nonlinear models, the situation is more complicated but when the health effects are 
small, as is almost surely the case with PM, the degree of bias is likely to be limited (Carroll et al., 1995; Thoresen and Laake, 2000; Thoresen, 2006). While we argue here that the exposure modeling plays the role of regression calibration, this leaves aside consideration of other sources of error such as the difference between ambient exposure and personal exposure and the one in three and one in six day sampling patterns of the monitors that likely introduce classical error.

Log scale. To assess the nature of the prediction (measurement) error using the validation information, we take $X$ to be the held-out PM observations, on the $\log$ scale, and $W$ and $S$ to be the predictions from our model (1), calculating $U=W-X$ and $V=X-S$ respectively under each measurement error model (S1-S2). We exclude the anomalous Pennsylvania site for $\mathrm{PM}_{10}$. There is less correlation (-0.07 for $\mathrm{PM}_{10}$ and -0.08 for $\mathrm{PM}_{2.5}$ ) between the predicted exposure, $S$, and the measurement error, $V=X-S$, assuming a Berkson structure, than between $X$ and $U$ (-0.51 and -0.40 for $\mathrm{PM}_{10}$ and $\mathrm{PM}_{2.5}$ respectively) assuming classical structure. This is more consistent with Berkson structure in which $S \perp V$. In addition, the variance of the predicted exposure, $\widehat{\operatorname{Var}}(S)$ (0.136 for $\mathrm{PM}_{10}$ and 0.118 for $\mathrm{PM}_{2.5}$ ) is lower than that of the held-out observations, $\widehat{\operatorname{Var}}(X)\left(0.183\right.$ for $\mathrm{PM}_{10}$ and 0.140 for $\left.\mathrm{PM}_{2.5}\right)$ as we would expect to result from smoothing, and consistent with Berkson structure with $\operatorname{Var}(X)=\operatorname{Var}(S)+\operatorname{Var}(V)$. Regressing the observations, $X$, on the predictions, $S$, we obtain an intercept of 0.15 for $\mathrm{PM}_{10}$ (0.11 for $\left.\mathrm{PM}_{2.5}\right)$ and a slope of 0.95 (0.96 for $\left.\mathrm{PM}_{2.5}\right)$, suggesting that a measurement error model of the form, $X=\gamma_{0}+\gamma_{1} S+V$, is more appropriate and that one might use $\hat{\gamma}_{0}+\hat{\gamma}_{1} S$ as the predicted exposure in the health modeling in place of $S=\hat{Y}$.

The prediction variances are reasonably homoscedastic, with a mean of 0.055 for $\mathrm{PM}_{10}$ (0.023 for $\left.\mathrm{PM}_{2.5}\right)$ and 2.5th and 97.5th percentiles of 0.040 (0.015) and $0.078(0.039)$. There is evidence of only limited spatial autocorrelation in the prediction errors based on empirical semivariograms, with most of the error captured in the nugget. Temporal autocorrelation is similar to that of the residuals (Section 3.2.3 in the main paper). Having chosen Berkson structure as our working model for the prediction errors, we can consider the distribution of the cross-validated prediction errors; standard measurement error models assume a normal distribution for the errors. For both $\mathrm{PM}_{2.5}$ and $\mathrm{PM}_{10}$, the standardized errors (dividing by the prediction standard errors) on the validation set are long-tailed but are reasonably symmetric, with a $t$ distribution with about $5 \mathrm{df}$ best fitting the data based on ad hoc comparison of q-q plot fits. 
Original scale. These results suggest that health analyses that use the predictions on the log scale without adjustment for measurement error may perform reasonably, based on the approximate Berkson structure, homoscedasticity and symmetry of the errors. However, health models generally relate outcomes to PM on the original scale. Since standard epidemiological analysis considers the proxy directly without adjusting for measurement error, and since the lack of normality on the log scale argues against an assumption of lognormal measurement error, we might consider to what degree additive error on the original scale approximates the measurement error. Exponentiation induces a complicated distribution for the measurement error on the additive scale, $V_{i}^{*}=\exp \left(X_{i}\right)-\exp \left(S_{i}\right)$, with approximate prediction variance of $\exp \left(S_{i}\right)^{2} \hat{\operatorname{Var}}\left(S_{i}\right)$ from the delta method. Again using the validation set, Berkson error still seems reasonable, with less correlation (.10 and -.09 for $\mathrm{PM}_{10}$ and $\left.\mathrm{PM}_{2.5}\right)$ between $\exp (S)$ and $V^{*}$ than between $\exp (X)$ and $U^{*}=\exp (W)-\exp (X)$ (-0.53 for $\mathrm{PM}_{10}$ and -0.40 for $\left.\mathrm{PM}_{2.5}\right)$. Also, the variance of the predicted exposures, $\widehat{\operatorname{Var}}(\exp (S))$, is lower $(77$ and 19 for $\mathrm{PM}_{10}$ and $\mathrm{PM}_{2.5}$ ) than the variance of the held-out observations, $\widehat{\operatorname{Var}}(\exp (X))$ (107 for $\mathrm{PM}_{10}$ and 22 for $\mathrm{PM}_{2.5}$ ). After exponentiation, the errors no longer appear homoscedastic, with the prediction standard errors increasing linearly with the predicted values, as induced by the delta method approximation. The prediction variances are approximately five times as large for the largest predictions as for the smallest predictions. Regressing the observations on the predictions, again a measurement error model $\exp (X)=\gamma_{0}+\gamma_{1} \exp (S)+V^{*}$ seems most appropriate with intercepts of 2.2 and 0.76 for $\mathrm{PM}_{10}$ and $\mathrm{PM}_{2.5}$ respectively and slopes of 0.93 and 0.95 . There is little evidence for spatial autocorrelation, while the temporal autocorrelation is similar to that seen on the log scale. After exponentiation, the standardized residuals are skewed right, but the tail is not particularly long. These results suggest we might proceed in health analyses assuming an additive Berkson structure on the orginal scale, but that sensitivity analysis should consider heteroscedastic, skewed measurement error, possibly through a Bayesian approach.

Results concerning the measurement error implications for $\mathrm{PM}_{2.5}$ predictions for 1988-1998 are similar to the results for the core model. 


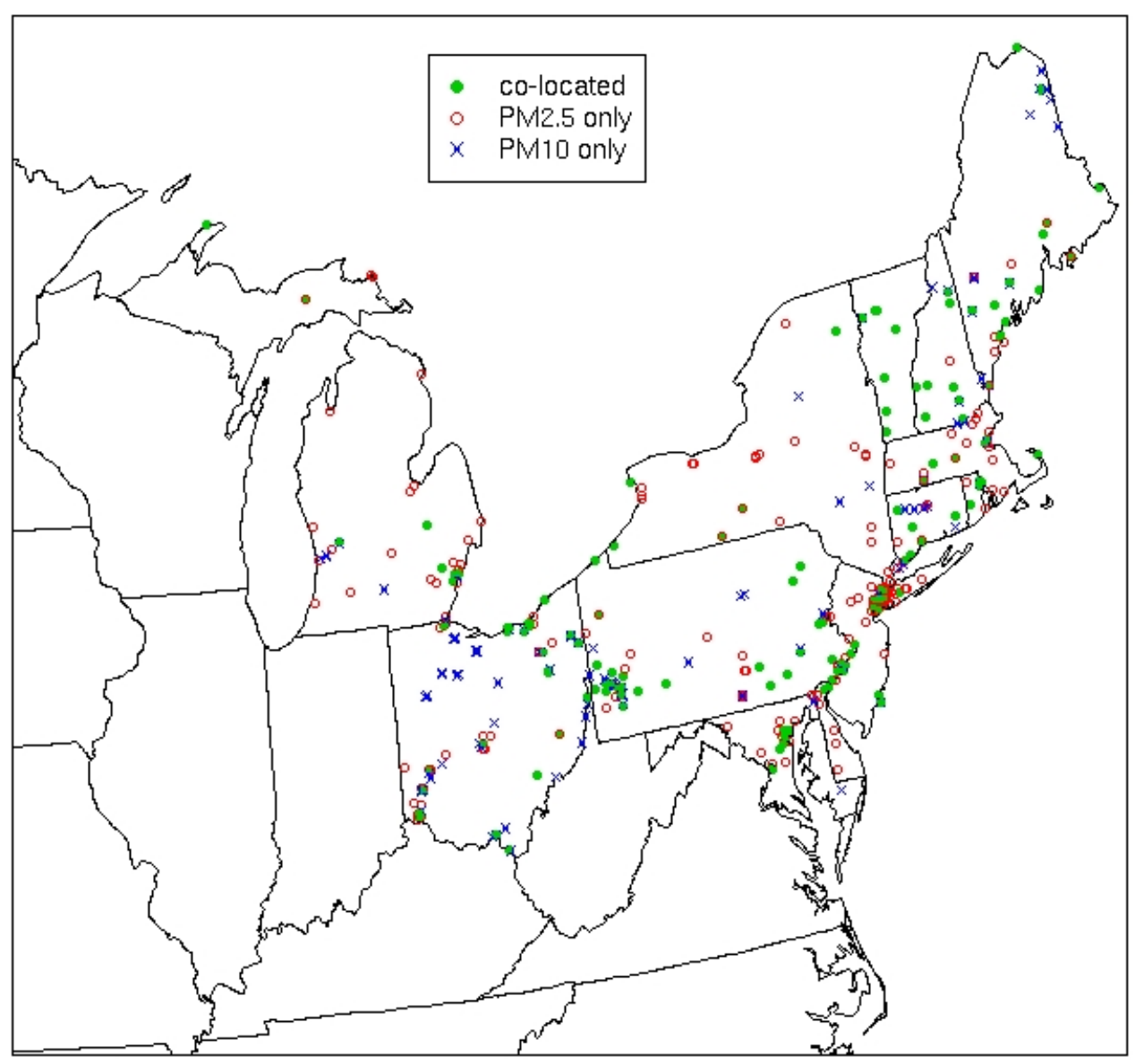

FIG S1. PM monitors for 1999-2002 reporting at least 12 months of data, split into colocated monitors and sites with only $P M_{2.5}$ or only $P M_{10}$ monitors. 


\section{References.}

R.J. Carroll, D. Ruppert, and L.A. Stefanski. Measurement Error in Nonlinear Models. Chapman \& Hall CRC, Boca Raton, Florida, 1995.

A. Gryparis, C.J. Paciorek, A. Zeka, J. Schwartz, and B.A. Coull. Measurement error caused by spatial misalignment in environmental epidemiology. Biostatistics, in press, 2008.

D. Ruppert, M.P. Wand, and R.J. Carroll. Semiparametric Regression. Cambridge University Press, Cambridge, U.K., 2003.

M. Thoresen. Correction for measurement error in multiple logistic regression: A simulation study. Journal of Statistical Computation and Simulation, 76:475-487, 2006.

M. Thoresen and P. Laake. A simulation study of measurement error correction methods in logistic regression. Biometrics, 56:868-872, 2000.

S.N. Wood. Generalized Additive Models: An Introduction with R. Chapman \& Hall, Boca Raton, 2006.

Christopher J. Paciorek

Department of Biostatistics

Harvard School of Public Health

Boston, Massachusetts 02115

USA

E-MAIL: paciorek@alumni.cmu.edu

URL: http://www.hsph.harvard.edu/ paciorek

Robin C. Puett

Cancer Prevention and Control Program

Department of Epidemiology and Biostatistics and

Department of Environmental Health Sciences

Arnold School of Public Health

University of South Carolina

Columbia, South Carolina 29208

USA

E-MAIL: rpuett@gwm.sc.edu
JEFF D. YANOSKY

HELEN H. SuH

Department of Environmental Health

Harvard School of Public Health

Boston, Massachusetts 02115

USA

E-MAIL: jyanosky@hsph.harvard.edu

E-MAIL: hsuh@hsph.harvard.edu

Francine LADEN

CHANNing LABORATORY

Brigham and Women's Hospital and Harvard Medical School Boston, Massachusetts 02215

AND

Departments of Environmental Health and Epidemiology Harvard School of Public Health

Boston, Massachusetts 02115

USA

E-MAIL: francine.laden@channing.harvard.edu 